\title{
Hypocalcified autosomal recessive amelogenesis imperfecta-A case report
}

\author{
Ali Azhar Dawasaz ${ }^{1}$, M. Zakirulla ${ }^{2}$, Meer Allahbaksh ${ }^{3}$ \\ ${ }^{1}$ Department MDS, College of Dentistry, King Khalid University, Abha, KSA \\ ${ }^{2}$ Department of Pediatric Dentistry, College of Dentistry, King Khalid University, Abha, KSA \\ ${ }^{3}$ Department of Public Health, Anglia Ruskin University, London, UK \\ Email:_draliazhar@gmail.com
}

Received 5 September 2012; revised 13 October 2012; accepted 20 October 2012

\begin{abstract}
Amelogenesis imperfecta is an unusual hereditory disorder affecting both primary and permanent dentition. We present a rare case of hypocalcified autosomal recessive amelogenesis imperfecta occuring in primary dentition in a 7-year-old girl with a family history of consanguineous marriage. Clinical and radiological examination revealed discoloration and hypoplasia of enamel with easy chipping affecting both maxillary and mandibular dentition.
\end{abstract}

Keywords: Hypocalcified; Autosomal Recessive; Consanguineous; Discoloration

\section{INTRODUCTION}

Amelogenesis imperfect (AI) encompasses a heterogeneous group of developmental disorders that demonstrate alterations in the enamel. AI is caused by mutations in genes that control amelogenesis and follows inheritance patterns of autosomal-dominant, autosomal recessive or $\mathrm{X}$-linked modes of transmission [1]. The enamel may be hypoplastic, hypomineralised or both, and teeth affected may be discoloured, sensitive or prone to disintegration either post eruption (post-eruptive breakdown) or preeruption (idiopathic resorption) [2].

Amelogenesis imperfecta (AI) is a group of conditions, genetic in origin, which affect the structure and clinical appearance of the enamel of all or nearly all the teeth [3]. AI encompasses a complicated group of conditions that demonstrate developmental alterations in the structure of the enamel in the absence of a systemic disorder [4]. This enamel anomaly affects both primary and permanent dentition. The incidence of AI has been reported as varying between $1: 700$ and 1:16,000, depending on the diagnostic criteria and the population studied [5]. Hypoplastic AI represents $60 \%-73 \%$ of all cases, hypomaturation AI represents $20 \%-40 \%$, and hypocalcification AI represents 7\% [6]. Weinmann et al., 1945, subdi- vided amelogenesis imperfecta into hypoplastic and hypocalcified types [7]. Several classifications have evolved since then, with at least ten subtypes, characterized by clinical features and mode of inheritance. Two X-linked phenotypic variants of amelogenesis imperfecta have been included in these classifications-a hypoplastic form and a hypomaturation form. Witkop and Sauk listed the varieties of AI, divided according to whether the abnormality lay in a reduced amount of enamel (hypoplasia), deficient calcification (hypocalcification), or imperfecta maturation of the enamel (hypomaturation), and also recognized the combined defects [3]. The appearance depends on the type of AI, varying from the mild hypomature "snow-capped" enamel to the more severe hereditary hypoplasia with thin, hard enamel which has a yellow-brown appearance. Dental problems, which depend on the severity of the condition, include sensitive teeth and poor appearance due to tooth loss and staining. If tooth tissue loss is severe there is vertical loss resulting in reduced masticatory function and poor appearance. Pulpal involvement may occur in severe cases, and because the roughness makes cleaning more difficult, gingivitis and periodontitis may develop. As AI affects both primary and permanent dentition, prevention of future dental problems must start from the beginning, by educating the parents and reviewing the patient regularly. Attention must also be paid to the psychological health of the patient [8].

\section{CASE REPORT}

A 7-year-old female patient presented with the chief complaint of loose upper front teeth and wanted to extract them. She had also difficulty in chewing with her posterior teeth as the teeth had become small and used to easily chip off. A detailed medical, dental, and social history was obtained. Family history revealed that her parents had a consanguineous marriage. Her siblings were normal with healthy teeth. 
The enamel of all the teeth was hypoplastic and yellow-brown in color. The surfaces of the teeth were smooth, and the enamel was either not visible or very thin over the crowns of all teeth. Exposed dentin was brown in color. Apart from this, her past medical history was noncontributory.

On intraoral examination, it was found that she had mobile maxillary right primary central incisor. The thickness of enamel was reduced and was completely chipped off from some teeth exposing the dentin. The teeth, in general, exhibited a yellowish brown discoloration (Figures 1 and 2). There was a localized area of pitting present on the exposed labial tooth surface of maxillary left primary lateral incisor \& open bite was present (Figures 3 and 4). Examination of the periodontium revealed the presence of generalized, marginal, and papillary gingivitis, with calculus deposition and unsatisfactory oral hygiene.

Radiographic investigations included an orthopantomogram (OPG). The OPG showed the mixed dentition period with presence of erupting mandibular permanent central incisors. Pulp chambers were of normal size and shape. The generalized lamina dura were distinct and normal (Figure 5). The diagnosis of hypocalcified, rough, autosomal recessive AI was confirmed on the basis of family history, clinical and radiographic features. Esthetics along with functional limitations was the reason the patient's parents brought her to the hospital for treatment. The treatment proposed for her ranged from extraction of maxillary right primary central incisor to oral prophylaxis and resin bonded restorations for posterior teeth.

\section{DISCUSSION}

Amelogenesis imperfecta is a developmental, often inherited disorder, affecting dental enamel. It usually occurs in the absence of systemic features and comprises of diverse phenotypic entities. The predominant clinical manifestations of affected individuals are enamel hypoplasia (enamel is seemingly correctly mineralized, but

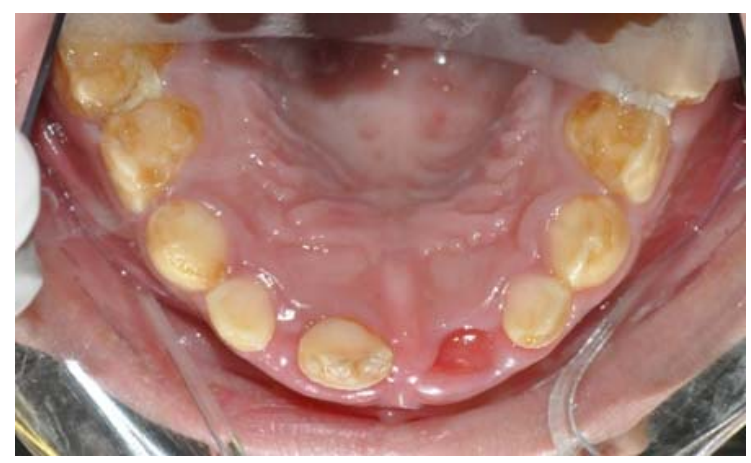

Figure 1. Multiple brownish discoloration of maxillary primary dentition.

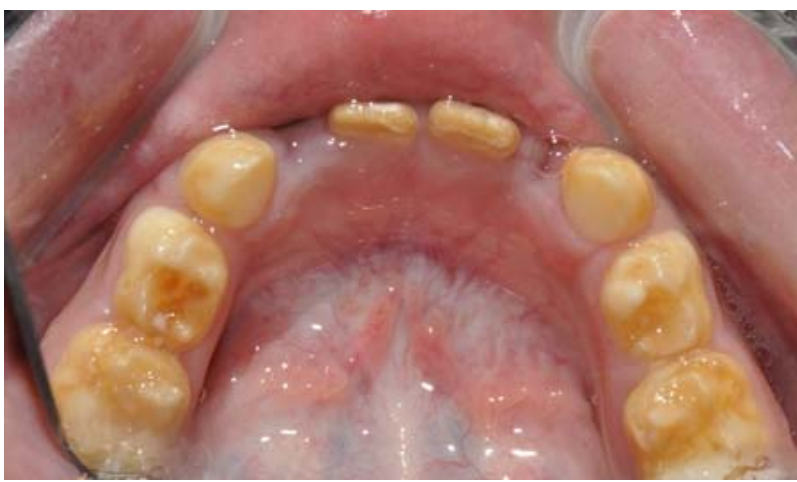

Figure 2. Multiple brownish discoloration of mandibular primary dentition.

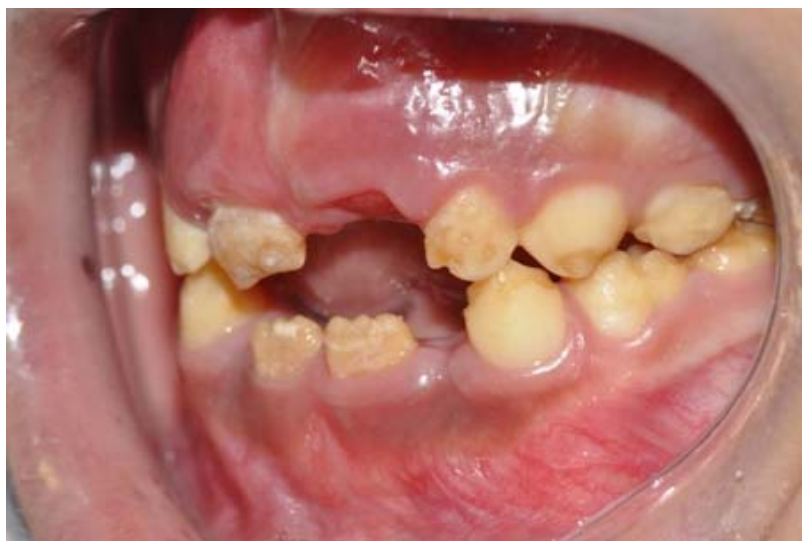

Figure 3. Multiple pits present on the labial surface of maxillary left primary lateral incisor mandibular primary dentition.

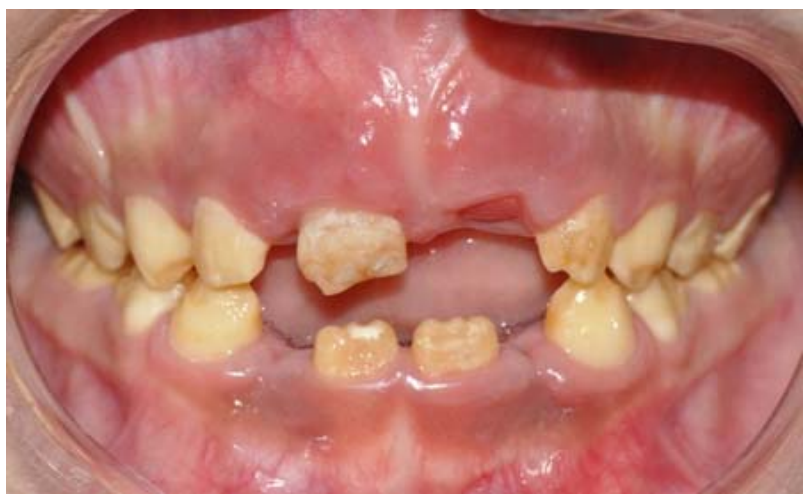

Figure 4. Anterior open bite present.

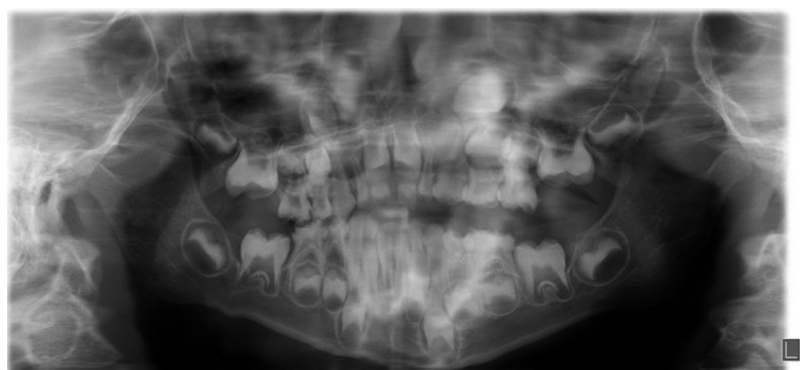

Figure 5. OPG showing generalized thinning of enamel. 
thin), hypomineralization (subdivided into hypomaturation and hyocalcification), or a combined phenotype, which is seen in most cases [9]. The trait of AI can be transmitted by an autosomal-dominant, autosomal-recessive, or X-linked mode of inheritance [10].

Approximately 7\% of individuals affected by amelogenesis imperfecta have the hypocalcified variant [11]. Hypocalcified AI is thought to result from a defect in initial crystallite formation followed by defective growth [8]. The enamel is also insufficiently mineralized, extremely soft and may show a chalky, dull color or a cheesy consistency with the possibility of a rapidly break down. These teeth have an abnormal shape when they erupt, an abnormal color and dull appearance. Loss of enamel from wear and staining tends to increase with age. Interestingly, the enamel at the cervical portion is frequently better calcified than that on the rest of the crown [12]. Successful management of AI during childhood requires the cooperation and motivation of the patient and parents.

Autosomal recessive AI (ARAI) was diagnosed considering the history of consanguinity in patient's family. This may be more often encountered in certain ethnic and cultural groups where intermarriage within the family may be more common [13]. ARAI will also be more prevalent where there is a high frequency of the mutant gene in a population, such as in some Polynesian communities [14].

Amelogenesis imperfecta cases necessitate careful diagnoses to improve function and esthetics because they present with a complex set of problems, such as decreased occlusal vertical height, deep bite, rampant caries attributable to plaque accumulation, abnormalities in dental eruption, tooth sensitivity, and psychosocial problems related to poor esthetics [15]. However, the restoration of esthetics and function of teeth in childhood patients suffering from amelogenesis imperfecta often represents to the dentist a major challenge [16].

Clinically, a skeletal anterior open bite is seen in approximately $50 \%$ of patients with AI of either X-linked or autosomal inheritance. Such an association might be regarded as a syndrome but this does not appear as such in any classification. The significance of this common association has yet to be elucidated [17].

Treatment is as ever based on the principles of prevention before intervention. However, in these patients' cases, intervention will likely be earlier and more radical than for other normal individuals. The progression of treatment during childhood has been described as a temporary phase followed by a transitory phase [18]. ARAI shows soft enamel due to a defect during mineralization and wear is common [19]. The primary dentition is protected by the use of preformed metal crowns on posterior teeth. Either polycarbonate crowns or composite restora- tions are used on anterior teeth [20]. The pretreatment with sodium hypochlorite can improve bond strength to hypocalcified enamel [19] but if just affected enamel is present it is advisable to remove it and bonding should be tried in dentin, usually sclerotic [21]. In order to achieve good bonding strength extended etching periods have been recommended for conventional adhesive systems [22]. As stated by Andrews et al. in most severe cases porcelain veneers appear to be the best option. When there is a greater loss of tooth structure, indirect restorations may provide better mechanical properties either to the tooth or to the restoration [23].

Children with AI are not without malocclusions and it is important that a restorative dentist and an orthodontist are involved with the pediatric dentist in the care plan from the child's early age. The anterior open bite seen in some cases of AI requires consideration of surgical as well as restorative management as seen in our case [2].

There are a great number of alternatives for the treatment of Hypocalcified AI teeth. This communication only state available options for the management of AI in children and helps the clinician to understand what can be used on each case. Analyzing the benefits and limitations of each technique the professional will be able to decide the best treatment plan.

\section{CONCLUSIONS}

A case report was presented describing the clinical and radiological features of a patient with a hypoplastic, autosomal recessive amelogenesis imperfecta. An awareness of the clinical features of amelogenesis imperfecta helps with identifying the condition and leading to successful treatment.

Even though AI is by definition a disorder of enamel, it has been associated with several other dental anomalies including disturbances in eruption, congenitally missing teeth, anterior open bite (AOB), pulpal calcifications, pathologic root and crown resorption, and taurodontism. Successful management of AI requires early recognition, preventive counseling, emotional support, and individualized restorative care adapted to the phases of tooth development. The psychosocial effects of AI on affected individuals, even in children are significant.

\section{REFERENCES}

[1] Witkop Jr., C.J. (1998) Amelogenesis imperfecta, dentinogenesis imperfecta and dentin dysplasia revisited: Problems in classification. Journal of Oral Pathology \& Medicine, 17, 547-553. doi:10.1111/j.1600-0714.1988.tb01332.x

[2] Crawford, P.J.M., Aldred, M. and Bloch-Zupan, A. (2007) Amelogenesis imperfect. Orphanet Journal of Rare Diseases, 2, 1-11. doi:10.1186/1750-1172-2-1 
[3] Aldred, M.J., Savarirayan, R. and Crawford, P.J. (2003) Amelogenesis imperfecta: A classification and catalogue for the 21st century. Oral Diseases, 9, 19-23. doi:10.1034/j.1601-0825.2003.00843.x

[4] Neville, B.W., Damm, D.D., Allen, C.M. and Bouquot, J.E. (2005) Abnormalities of teeth. In: Oral and Maxillofacial Pathology, 2nd Edition, Saunders, Philadelphia, 2005, 89.

[5] Rao, S. and Witkop Jr., C.J. (1971) Inherited defects in tooth structure. Birth Defects Original Article Series, 7, 153-184.

[6] Rajendran, R. (2007) Chapter 1. Developmental disturbances of oral and paraoral structures. In: Rajendran, R. and Sivapathasundhram, B., Eds., Shafer's Textbook of Oral Pathology, 5th Edition, Elsevier, Amsterdam, 2007, 67.

[7] Weinmann, J.P., Svobcda, J.F. and Woods, R.W. (1945) Hereditary disturbances of enamel formation and calcification. Journal of American Dental Association, 32, 397418.

[8] Coffield, K.D., Phillips, C., Brady, M., Roberts, M.W., Strauss, R.P. and Wright, J.T. (2005) The psychological impact of developmental dental defects in people with hereditary amelogenesis imperfecta. Journal of the American Dental Association, 136, 620-630.

[9] Backman, B. and Holmgren, G. (1988) Amelogenesis imperfecta: A genetic study. Human Heredity, 38, 189206. doi:10.1159/000153785

[10] Aldred, M.J. and Crawford, P.J. (1995) Amelogenesis imperfecta-Towards a new classification. Oral Diseases, 1, 2-5. doi:10.1111/j.1601-0825.1995.tb00148.x

[11] Bsoul, S.A., Flint, D.J., Terezhalmy, G.T. and Moore, W.S. (2004) Amelogenesis imperfecta. Quintessence International, 35, 338-339.

[12] Akin, H., Tasveren, S. and Yeler, D.Y. (2007) Interdisciplinary approach to treating a patient with amelogenesis imperfecta: A clinical report. Journal of Esthetic and Restorative Dentistry, 19, 131-135. doi:10.1111/j.1708-8240.2007.00083.x

[13] Michaelides, M., Bloch-Zupan, A., Holder, G.E., Hunt, D.M. and Moore, A.T. (2004) An autosomal recessive cone-rod dystrophy associated with amelogenesis imperfecta. Journal of Medical Genetics, 41, 468-473. doi:10.1136/jmg.2003.015792

[14] Crooks, M.C. (1990) Prevalence of developmental defects of enamel in children and young adults in the Cook Islands. New Zealand Dental Journal, 86, 39-41.

[15] Seow, W.K. (1993) Clinical diagnosis and management strategies of amelogenesis imperfecta variants. Pediatric Dentistry, 15, 384-393.

[16] Neto, N.L., Paschoal, M.A.B., Kobayashi, T.Y., Rios, D. and Silva, S.M.B. (2010) Early oral rehabilitation of a child with amelogenesis imperfect. Journal of the Health Sciences Institute, 28, 246-248.

[17] Yigit Ozer, S.G. and Bahsi, E. (2010) Treatment of an amelogenesis imperfecta with restorations prepared using a modified clear matrix technique. Journal of Investigative and Clinical Dentistry, 1, 59-63. doi:10.1111/j.2041-1626.2010.00007.x

[18] Bouvier, D., Duprez, J.P. and Bois, D. (1996) Rehabilitation of young patients with amelogenesis imperfecta: A report of two cases. ASDC Journal of Dentistry Child, $\mathbf{6 3}$ 443-447.

[19] Venezie, R.D., Vadiakas, G., Christensen, J.R. and Wright, J.T. (1994) Enamel pretreatment with sodium hypochlorite to enhance bonding in hypocalcified amelogenesis imperfecta: Case report and SEM analysis. Pediatric Dentistry, 16, 433-436.

[20] Sapir, S. and Shapira, J. (2001) Dentinogenesis imperfecta: An early treatment strategy. Pediatric Dentistry, 23, 232-237.

[21] Tay, F.R. and Pashley, D.H. (2004) Resin bonding to cervical sclerotic dentin: A review. Journal of Dentistry, 32, 173-196. doi:10.1016/j.jdent.2003.10.009

[22] Lopes, G.C., Vieira, L.C., Monteiro Jr., S., Caldeira de Andrada, M.A. and Baratieri, C.M. (2003) Dentin bonding: Effect of degree of mineralization and acid etching time. Operative Dentistry, 28, 429-439.

[23] Andrews, P., Levine, N., Milnes, A., Pulver, F., Sigal, M. and Titley, K. (1992) Advances in the treatment of acquired and developmental defects of hard dental tissues. Current Opinion in Dentistry, 2, 66-71. 